\title{
Successful Packet Reception Analysis in Multi-Packet Reception Wireless Systems
}

\author{
António Furtado, Student Member, IEEE, Rodolfo Oliveira, Senior Member, IEEE, \\ Rui Dinis, Senior Member, IEEE, and Luís Bernardo, Member, IEEE
}

\begin{abstract}
In this letter, we analyze the individual probability of receiving a packet when $n$ nodes transmit simultaneously to a single receiver and a multi-packet reception (MPR) scheme is adopted at the physical layer. The main contribution of this letter is the characterization of the average number of packets successfully received. The generic methodology proposed to compute the probability of successful reception of a packet is obtained taking into account the stochastic nature of the path loss due to the spatial distribution of the nodes, as well as shadowing and fast fading effects. The accuracy of the theoretical approach is finally assessed through simulations, showing that for a generic MPR system, there is an optimal number of transmitters that maximize the average number of received packets.
\end{abstract}

Index Terms-Multi-packet reception, wireless systems, performance evaluation.

\section{INTRODUCTION}

$\mathbf{N}$ OWADAYS, Infrastructure-Less Wireless Networks (ILWNs), which include ad hoc and sensor networks, are gaining increasing popularity as they do not need a fixed infrastructure. However, the capacity of ILWNs can be surprisingly low [1] for Single-Packet Reception (SPR) schemes, where any receiver can successfully decode at most one packet at a time. Recently, a few works have shown that the capacity of ILWNs can be improved [2] if MultiPacket Reception (MPR) schemes are adopted, where multiple packets transmitted at the same time from different sources are decoded at the receiver.

In this work we propose a new methodology to compute the individual probability of successful reception in MPR schemes as well as the average number of received packets when $n$ simultaneous transmissions occur. The proposed approach is based on the generic receiving condition presented in [3] and [4], in which the reception of a packet succeeds if the received power that defines the Signal-to-Interferenceplus-Noise-Ratio (SINR) is higher than a certain receiving threshold.

As the main contribution of our work, the proposed solution is formulated in a generic way, being valid for any spatial distribution of the transmitters and any propagation model,

Manuscript received June 21, 2016; revised August 17, 0000; accepted August 29, 2016. Date of publication September 5, 2016; date of current version December 8, 2016. This work was partially supported by the Portuguese Science and Technology Foundation (FCT/MEC) under the Project UID/EEA/50008/2013 and in part the studentship SFRH/BD/88140/2012. The associate editor coordinating the review of this letter and approving it for publication was P. Serrano.

The authors are with the Instituto de Telecomunicações, 1049-001 Lisbon, Portugal, and also with the Centre of Technology and Systems, Departmento de Engineering Electrotécnica, Faculdade de Ciências e Tecnologia, Universidade Nova de Lisboa, 2829-516 Caparica, Portugal.

Digital Object Identifier 10.1109/LCOMM.2016.2606105 as long as the received signals from the multiple transmitters are independent and identically distributed (i.i.d.). A generic noise distribution is also considered at the receiver side. The computation time of the proposed solution is limited by the algorithm adopted to compute the Fourier transform and it is independent of the number of simultaneous transmissions. This is one of the main advantages of the proposed solution. Our technique allows the computation of optimal number of transmitters for a given reception threshold, which is also an important point in the design of medium access control (MAC) techniques for MPR systems. The probability of successful packet reception is derived in Section II. Section III describes a typical scenario of application. Section IV evaluates the proposed methodology and some concluding remarks are presented in Section V.

\section{SySTEM MODEL}

In this work we consider a network where $n$ nodes transmit data simultaneously to a single receiver. It is assumed that the signals received from the transmitters are i.i.d. random variables (RVs), characterized by the Probability Density Function (PDF) $f_{P_{k}}$. The total power received from the multiple transmissions is expressed by

$$
\Lambda=\sum_{k=1}^{n} P_{k}+N_{0},
$$

where $P_{k}$ is a random variable representing the power received from the k-th transmitter and $N_{0}$ is a RV representing the noise power at the receiver.

We assume an MPR receiver where the Signal-toInterference-plus-Noise ratio (SINR) associated to the signal received from node $j$ is defined by

$$
\gamma_{j}=P_{j} /\left(\Lambda-P_{j}\right) \text {. }
$$

Following the capture condition defined in [3], the signal from $j$ is successfully received if the following condition holds

$$
\gamma_{j}>b \text {. }
$$

The receiving threshold $b$ is a parameter determined by several factors such as the type of modulation and sensitivity of the receiver. Typically for SPR systems $b$ is between 1 and 10, and for MPR systems $b<1$ [5] (for example if (3) is used to model a CDMA system, a lower $b$ may represent a higher spreading factor and vice versa).

Departing from (3) and using (2), the necessary condition for successful reception of the signal from $j$ is given by

$$
P_{j}>\Lambda b /(b+1),
$$

which may be rewritten as $P_{j}>b^{\prime} \Lambda, b^{\prime}=b /(b+1)$. From (4), the probability of successful reception may be 